\title{
Vezikoüreteral Reflüde Antibiyotik Profilaksisi
}

\author{
Antibiotic Prophylaxis in Vesicoureteral Reflux
}

Onur Telli ${ }^{1}$ Barıș Esen ${ }^{1}$, Tarkan Soygür1', Berk Burgu' ${ }^{1}$

Ankara Universitesi Tıp Fakuiltesi Üroloji Anabilim Dalı Çocuk Ürolojisi Bilim Dalı
Geliș tarihi : 29.06.2015• Kabul tarihi: 22.10.2015

İletișim

Uz. Dr. Onur Telli

Tel: 03125955564

E-posta: onurtelli@yahoo.com

Ankara Üniversitesi Tıp Fakültesi Cebeci Araștırma ve

Uygulama Hastanesi Üroloji Anabilim Dalı çocuk Ürolojisi Bilim Dalı 06590 Cebeci/Ankara

Primer vezikoüreteral reflü (VUR) mesane disfonksiyonu ve üriner sistem enfeksiyonu riskinde artıșla yakından ilișkili olan çocukluk çağına ait sık ve önemli bir patolojidir. VUR tanısı alan çocukların çoğunda kendiliğinden geçse de, bir kısmında reflü nefropatisi, hipertansiyon, idrar yolu enfeksiyonu ve mesane disfonksiyonu gibi olumsuz sonuçlar doğurabilmektedir. Antibiyotik profilaksisi VUR'lu hastalarda ilk tedavi seçeneği olarak kabul edilirken son çalıșmalara bakıldığında kesin bir görüș birliği olmadığı izlenmektedir. Bu derlemede primer VUR'de antibiyotik profilaksisine güncel yaklașımlar güncel kılavuzlar eșliğinde değerlendirilmiștir.

Anahtar Sözcükler: Vezikoüreteral Reflü, Pprofilaksi, Antibiyotik

Vesicoureteral reflux (VUR) which is associated with bladder dysfunction and increased risk of urinary tract infection (UTI), is a common and important urinary system abnormality seen in children. Although VUR spontaneously resolves in most of the cases, it might lead to severe complications including reflux nephropathy, hypertension, UTI and bladder dysfunction. The continuous antibiotic prophylaxis (CAP) in the management of VUR is first choice of therapy. However, controversy exists on CAP in the current studies and some questions have not been properly answered yet. In this paper the recent trends of CAP in VUR is reviewed under current guidelines.

Key Words: Vesicoureteral Reflux, Prophylaxis, Antibiotic

Primer vezikoüreteral reflü (VUR), idrarin mesaneden üretere retrograd ak1mı olarak tanımlanmaktadır (1). Sağliklı hasta popülasyonunda VUR insidans1 $<\% 1$ iken, ateşli idrar yolu enfeksiyonu geçiren hastalarda bu insidans çok daha yüksektir. Bu durum, VUR'un rekürren idrar yolu enfeksiyonlarına (IYE), pyeloneritlere ve buna bağlı olarak renal skar oluşumuna neden olduğunu düşündürmüştür (2).

VUR tedavisinde temel amaç pyelonefrit riskini azaltarak renal fonksiyonu mümkün olduğunca korumaktır. Reflünün spontan düzelmeye dogă bir yatkınlığ vardır. Büyümeyle birlikte submukozal üreterin boyu uzar, üreterovezikal bilesske yeniden s,ekillenip antireflü mekanizma saglamlaşır ve mesane dinamikleri düzelir. VUR tedavisinde günümüzdeki tedavi seçenekleri; cerrahi tedaviler ya da antibiyotik profilaksi verilmesidir. Konservatif yaklaşım gözlem (yalnızca IYE geçirdiğnde IYE tedavisi), intermit$\tan$ ya da devaml antibiyotik profilaksisini (DAP) ve alt üriner sistem disfonksiyonu olanlarda mesane rehabilitasyonunu içeririr. Konstipasyon diyeti, hidrasyon, zamanlı defekasyon ve düzenli işeme ile IYE sıklı̆̆1 azalır; reflünün düzelmesine yardimc1 olur. VUR'de antibiyotik profilaksi verilmesinin amac1; idrarı steril tutarak pyelonefrit riskini azaltmak ve pyelonefrite sekonder yeni renal skar oluşumunun önüne geçmektir. Literatürde antibiyotik profilaksisinin koruyucu etkisi üzerine farklı hasta popülasyonlarında yapılmış ve birbirinden farklı sonuçlar bulunmuş randomize kontrollü çalışmalar bulunmaktadır. Bu derlemede, antibiyotik profilaksisi verilmesinin VUR'da rekürren IYE gelişimi ve yeni renal skar oluşumu üzerine koruyucu etkisi üzerine son 10 yll içerisinde yapılmış randomize kontrollü çalışmalar sistematik bir şekilde derlenmiştir. 


\section{YAKIN ZAMANDA DEVAMLI ANTIBIYYOTIK TEDAVISi ÜZERINE YAPILAN ÇALIȘMALAR:}

DAP ana problemler; etkinliğini gösteren kanıtların olmadığına dair kaygılar, hastanın ilaç kullanımına uyumunun k1sıtlı olduğuna dair yapılan bazı çalişmalar, ve bu tedavinin antibiyotik dirençli organizmaların ortaya çıkmasına neden olabileceğine dair kaygılar öne çıkmaktadır (3).

Son 10 yıl içerisinde VUR' da antibiyotik tedavisinin etkinliğini sorgulayan ve farklı sonuçlar bulan çeşitli çok merkezli çalışmalar yapılmıştır. Tablo 1 bu çalışmaları özetlemekte ve bu çalışmalarda alınan hasta popülasyonun özellikleri ve her kriterde buldukları sonuçlardaki farklılıklar vurgulanmaktadir.

VUR' da antibiyotik profilaksisini başar1sız bulan çalışmalara bakıldığında görece daha düşük riskli; düşük dereceli reflüsü olan, daha önce sadece $1 \mathrm{kez}$ idrar yolu enfeksiyonu geçirmiş, ve eğer araştırılmışsa düşük renal hasarı olan hastaların çalışmaya dahil edildi- ği görülmektedir. IYE tanısı konulmasında kullanılan "poşet" spesmenler kullanılması ve erkek çocukların sünnetsiz olması ya da sünnet durumunun bilinmiyor olması, artan yüksek IYE oranlarına katkıda bulunmuş olabilir.

Roussey-Kesler ve ark.(4) tarafinca yapılan bir çalışmada, antibiyotik profilaksisini başarısız bulan çalışmaların sonuçlarını da sorgulamak amaciyla düşük dereceli reflüsü olan (Grade I-III) 1 ay- 3 yaş arası ilk ateşli idrar yolu enfeksiyonu sonrası voiding sistoüretrografi (VCUG) ile tanı konan hastalar çalışmaya dahil edilmiştir. Bu çalışmada antibiyotik profilaksisi sadece grade III VUR olan erkek hastalarda enfeksiyon riskinde istatistiki olarak anlamlı bir azalmayı sağladığı gösterilmiștir. Bunun yanında Isvec, reflü çalışmasında, yüksek reflülü (Grade III-V) olan, rekürren IYE ve renal skar gelişim riski açısından kız ve erkek cinsiyetler arasındaki farka dikkat çekilmis,tir. Bir iki yas, arası II- IV derece refluilü 203 çocugun katıldı̆̈ bu çalışmada DAP, endoskopik enjeksiyon ve yalnızca gözlemin etkinliğ karş,las,tırılmıs,tır. DAP grubundaki 43 kız hastanın 8'inde (\% 19), enjeksiyon yapilan $43 \mathrm{k} 1 \mathrm{z}$ hastanin 10'unda (\% 23) ve gözlem grubundaki $42 \mathrm{k} 1 \mathrm{z}$ hastanın 24'ünde (\% 57) ateşli IYE gözlenmis,tir. DAP grubundaki kızlarda hic, yeni skar olus,umu görulmezken, endoskopi grubundaki 5 hastada ve gözlem grubundaki 8 hastada yeni skar tespit edilmis,tir. Erkeklerde ise IYE tekrarı veya renal hasar açısından bir farklilik bulunmamıştır (5). Daha önce en az bir kez ateşli IYE geçiren 576 hastanın alındığ 1 (Prevention of Recurrent Urinary Tract Infection in Children with Vesicoureteric Reflux and Normal Renal Tracts) PRIVENT çalışmasinda en az bir kez IYE geçiren 576 çocuk 12 ay boyunca günlük trimetoprim-sulfamethoksazol veya plasebo almak üzere iki gruba ayrılmış ve hastaların \%42'inde VUR izlenmiştir. Plasebo grubunda hastaların $\% 19$ 'unda ve trimetoprimsulfamethoksazol grubunda ise hastaların \%13'ünde IYYE geliştiği gözlenmiştir. Fakat antibiyotiklerin ateşli IYE gelişmesini önleyen bu etkisinin yaş, cinsiyet, reflü durumu ve reflü

Tablo 1: VUR' da antibiyotik tedavisinin etkinliğini sorgulayan ve farklı sonuçlar bulan çeşitli çok merkezli çalışmalar

\begin{tabular}{|c|c|c|c|c|c|c|c|c|}
\hline \multirow[b]{2}{*}{ Çalışmalar } & \multicolumn{4}{|c|}{ Antibiyotik profilaksisini başarısız bulan yayınlar } & \multicolumn{4}{|c|}{ Antibiyotik profilaksisini başarılı bulan yayınlar } \\
\hline & $\begin{array}{l}\text { Garin } \\
\text { et al (11) }\end{array}$ & \begin{tabular}{|l|}
$\begin{array}{l}\text { Montini } \\
\text { et al (7) }\end{array}$ \\
\end{tabular} & \begin{tabular}{|l|}
$\begin{array}{l}\text { Pennesi } \\
\text { et al (12) }\end{array}$ \\
\end{tabular} & $\begin{array}{l}\text { Hari } \\
\text { et al (13) }\end{array}$ & $\begin{array}{l}\text { Roussey-Kessler } \\
\text { et al (4) }\end{array}$ & \begin{tabular}{|l|}
$\begin{array}{l}\text { Swedish Reflux Trial } \\
\text { (Branström et al (5) }\end{array}$ \\
\end{tabular} & \begin{tabular}{|l} 
PRIVENT \\
Craig et al (6)
\end{tabular} & \begin{tabular}{|l|}
$\begin{array}{l}\text { RIVUR } \\
\text { (Hoberman et al) (8) }\end{array}$ \\
\end{tabular} \\
\hline Y1l & 2006 & 2008 & 2008 & 2014 & 2008 & 2010 & 2009 & 2014 \\
\hline Hasta yaşı & $\begin{array}{l}3 \text { ay- } \\
18 \text { y1l }\end{array}$ & 2 ay- $<7$ yaş & $<30$ ay & $1-12$ yaş & $\begin{array}{l}1 \text { ay- } \\
3 \text { y1l }\end{array}$ & $12-24$ ay & $<18$ yaş & 2 ay-6 y1l \\
\hline K1z/Erkek hasta sayısı & $128 / 75$ & $234 / 104$ & $52 / 48$ & $31 / 62$ & $156 / 69$ & $128 / 75$ & $369 / 207$ & $558 / 49$ \\
\hline Takip süresi & $1 \mathrm{y} 1 \mathrm{l}$ & $1 \mathrm{y} 1 \mathrm{l}$ & 2 y1l $\mathrm{Ab}, 4$ y1l takip & 5 y1l & $1.5 \mathrm{y} 11$ & 2 y1l & $1 \mathrm{y} 1 \mathrm{l}$ & 2 y1l \\
\hline VUR derecesi & 0 -III & 0 -III & III-IV & I-IV & I-III & III-IV & $0-\mathrm{V}$ & I-IV \\
\hline $\begin{array}{l}\text { Bazal renal skar varlığ } \% \\
\text { DAP(-) } \\
\operatorname{DAP}(+)\end{array}$ & $\begin{array}{l}3.4 \\
9\end{array}$ & $\begin{array}{l}27.5 \\
33.1\end{array}$ & $\begin{array}{l}18 \\
22\end{array}$ & $\begin{array}{l}13 \\
23.4\end{array}$ & $\begin{array}{l}\text { Bilgi yok } \\
\text { Bilgi yok }\end{array}$ & $\begin{array}{l}\begin{array}{l}57(\mathrm{k} 1 \mathrm{z}) \\
69(\text { erkek}) \\
51(\mathrm{k} 1 \mathrm{z}) \\
81(\text { erkek })\end{array} \\
\end{array}$ & $\begin{array}{l}25 \\
25\end{array}$ & $\begin{array}{l}3.1 \\
4.1\end{array}$ \\
\hline $\begin{array}{l}\text { Yeni Skar oluşumu \% } \\
\text { DAP(-) } \\
\text { DAP }(+)\end{array}$ & $\begin{array}{l}3.4 \\
9\end{array}$ & $\begin{array}{l}1.9 \\
1.1\end{array}$ & $\begin{array}{l}36 \\
40\end{array}$ & $\begin{array}{l}7 \\
10.8\end{array}$ & $\begin{array}{l}\text { Bilgi yok } \\
\text { Bilgi yok }\end{array}$ & $\begin{array}{l}19(\mathrm{k} 1 \mathrm{z}) \\
4(\text { erkek }) \\
0(\mathrm{k} 1 \mathrm{z}) \\
0(\text { erkek })\end{array}$ & $\begin{array}{l}8 \\
7\end{array}$ & $\begin{array}{l}8.4 \\
8.2\end{array}$ \\
\hline $\begin{array}{l}\text { Rekürren ateşli İYE \% } \\
\text { DAP(-) } \\
\text { DAP(+) }\end{array}$ & $\begin{array}{l}1.7 \\
12.9\end{array}$ & $\begin{array}{l}19.6 \\
12.1\end{array}$ & $\begin{array}{l}30 \\
36\end{array}$ & $\begin{array}{l}3 \\
10\end{array}$ & $\begin{array}{l}26 \\
13\end{array}$ & $\begin{array}{l}57(\mathrm{k} 1 \mathrm{z}) \\
4(\text { erkek }) \\
19(\mathrm{k} 1 \mathrm{z}) \\
8(\text { erkek })\end{array}$ & $\begin{array}{l}12.2 \\
20.6\end{array}$ & $\begin{array}{l}37.4^{*} \\
25.4^{* *} \\
\\
25.5^{*} \\
12.8^{* *} \\
\end{array}$ \\
\hline Odds Ratio & $\begin{array}{l}7.38 \\
(0.88-61.97)\end{array}$ & \begin{tabular}{|l|}
1.62 \\
$(0.24-1.64)$ \\
\end{tabular} & $\begin{array}{l}1.20 \\
(0.54-2.64) \\
\end{array}$ & 3.33 & $\begin{array}{l}0.81 \\
(0.38-1.72) \\
\end{array}$ & $\begin{array}{l}0.39 \\
(0.18-0.88)\end{array}$ & $\begin{array}{l}0.58 \\
(0.26-1.32) \\
\end{array}$ & $\begin{array}{l}0.54 \\
(0.35-0.82) \\
\end{array}$ \\
\hline
\end{tabular}

DAP, Devamlı antibiyotik Profilaksisi; VUR, Vezikoüreteral Reflü;

* 2 yıllık takipte kayıp verisi olup IYE geçirmiş Kabul edilenler

** 2 yıllık takiplerinde kayıp verisi olup IYE geçirmemiş kabul edilenler 
derecesi gibi değişkenlerden etkilenmediği ve yeni renal skar oluşumunda 2 grup arası bir fark olmadığ görülmüştür (6). Başka bir kontrollü randomize çalışmada ilk ateşli IYY sonrasi profilaksi uygulanmayan ve 12 ay boyunca profilaksi uygulanan iki grup karşılaştırılmış ve profilaksi almayan 46 hastanin 9'unda ve profilaksi alan 82 hastanın 10'unda tekrar eden IYYE izlenmiştir (7).

NIH (National Institute of Health) tarafinca yapılan çok merkezli, prospektif, randomize bir kontrollü bir çalışma olan RIVUR çalışması (Randomized Intervention for Children with Vesico-Ureteral Reflux) ile grade I-IV reflüsü olan hastalarda 2 ay- 6 yaş aralığındaki 607 hastada antibiyotik profilaksisinin etkinlğini araştırılmıştır. İki yıl takip süresi olan bu çalışmada ateşli idrar yolu enfeksiyonu birincil sonuç olarak değerlendirilirken, antibiyotik tedavisini birakma ve renal skar oluşumu ise ikincil sonuçlar olarak değerlendirilmiştir. Profilaksi grubunda 302 hasta düşük doz trimetoprim-sulfametaksozal (TMP-SMX) alırken, 305 hasta plasebo ilaç kullanılmıştır. İki yıl sonunda profilaksi grubunda rekürren ateşli enfeksiyon yüzde 50 daha az bulunurken, renal skar oluşumu oranları arası herhangi bir fark görülmemiştir. Antibiyotik profilaksisinin yararı özellikle mesane disfonksiyonu olanlarda daha belirgin olduğu görülmüş ve $\% 63$ oranında antibiyotik direnci ile karş̧lașllmıs,tır. Bu çalışma değerlendirilmesinin grade IV VUR olan hasta sayısının az olduğu, daha çok düşük grade reflüsü olan hastaların alındığı, ve de hastaların çoğunluğunun k1z olduğu (558/607) göz önünde tutulması gerekir (8). RIVUR ve diğer çalışmaların sonucunda IYE riski yuiksek oldugu için kız çocuklarında, spontan rezolüsyon oranı düş,ik olan yuiksek dereceli ve büyrik çocuklardaki refluilerde renal hasar ilerleyebileceğ için renal skarı olan VUR'lu çocuklarda DAP tercih edilebilir.

Yapılan randomize kontrollü çalışmalar da göstermektedir ki; hasta özellikleri, reflü derecesi ve eşlik eden alt üriner sistem disfonksiyonu gibi durumlar, profilaksi tedavisi ile enfeksiyon rekürrensi arasındaki sonuçların çok farklı çıkmasına neden olmaktadır. $\mathrm{Bu}$ da hekimin tedavi planlamasinda hastanın bireysel özelliklerini ve kendi deneyimlerini olası sonuçları aile ile tartışarak vermesinin önemini daha da arttırmaktadır.

\section{Kılavuzlar}

Literatürdeki verilerin genelde retrospektif ve düşük kanıt düzeyine sahip veriler olması ve birbiri ile çelişen sonuçlar ortaya koyması nedeni ile kesin hükümler ortaya koyan kilavuzlar ortaya koymak vezikoüreteral reflü açısindan zordur. Ancak hekimlere pratik bir yaklaşım geliştirmeleri açısından Amerikan Üroloji kilavuzları (AUA) ve Avrupa Üroloji kilavuzlar1nin (EAU) vezikoüreteral reflüde antibiyotik profilaksisi üzerine önerilerinden kısa bahsetmek yararlı olacaktir;

\section{EAU:}

EAU k1lavuzları antibiyotik profilaksisinin düşük reflü evreli hastalarda etkinliğinin olmadığı ya da çok kısıtlı etkinliği olduğunu söylemekle birlikte, grade III-IV reflüde profilaksinin rekürren enfeksiyonu engellediğini çalışmaların gösterdiği söyler ancak ileri renal hasar oluşumunu engellediği kanıtlanmadığını belirtir.

Antibiyotik profilaksisi gerekmeyen hastaları seçmek risklidir. Karar vermede genç yaş, alt üriner sistem disfonksiyonu varlı̆̆1, kız cinsiyet ve sünnet durumu gibi IYE için risk faktörleri varlığ1 etkili olabilir. Tuvalet eğitimi tamamlanıp alt üriner sistem disfonksiyonu olmadığ gösterilinceye kadar tüm reflülü çocuklara antibiyotik profilaksisi verilmesini hekimler açısından pratik bir yaklaşım olarak önerir. Ayrıca alt üriner sistem disfonksiyonu olan tüm çocuklara mutlaka verilmesini önerir (9).

\section{AUA:}

AUA kılavuzları <1 yaş, grade I-II reflüsü olan, geçirilmiş ateşli IYYE olmayan ve renal kortikal anomalisi olmayan hastalarda antibiyotik profilaksisini uygun bir seçenek olarak vermektedir. Bir yaş üstü çocuklarda ise ateşli IYE öyküsü olmayan, mesanebağırsak disfonksiyonu olmayan ve renal kortikal anomalisi olmayan hastalar için profilaksi verilmeden takip edilmesi uygun bir seçenek olarak verilmektedir (10).

\section{SONUÇ}

Literatürde yapılmış olan randomize kontrollü çalışmalar farklı hasta popülasyonları alınarak yapılması nedeniyle antibiyotik profilaksisinde kesin hükümler vermeye yetecek kanıt ortaya koyamamaktadır. Kilavuzlarda da belirttiği üzere; özellikle alt üriner sistem disfonksiyonu olan ve yüksek dereceli reflüsü olan hastalarda antibiyotik profilaksisinin rekürren idrar yolu enfeksiyonu riski azaldığ1 çalışmalarda görülmesine rağmen düşük dereceli reflüsü olan hastalarda rekürren IYE üzerine ortaya konan çalışmalar tartışmalıdır. Antibiyotik profilaksisinin etkinliği üzerine daha yüksek kanıt düzeyli bilgiler ortaya konabilmesi için daha geniş ve homojen hasta popülasyonlarının alındığı ek prospektif randomize çalışmalara ihtiyaç vardır. 


\section{KAYNAKLAR}

1. Lopez PJ, Celis S, Reed F, et al. Vesicoureteral reflux: current management in children. Curr Urol Rep. 2014;15: 447.

2. Hollowell JG, Greenfield SP . Screening siblings for vesicoureteral reflux . J Urol 2002; 168: 2138 - 41 .

3. Baquerizo BV, Peters CA. Antibiotic prophylaxis and reflux: critical review and assessment. F1000Prime Rep. 2014; 6: 104.

4. Roussey-Kesler G, Gadjos V, Idres N, et al. Antibiotic prophylaxis for the prevention of recurrent urinary tract infection in children with low grade vesicoureteral reflux: results from a prospective randomized study. J Urol. 2008; 179: 674-9.

5. Brandström P, Esbjörner E, Herthelius $\mathrm{M}$, et al. The Swedish reflux trial in children: I. Study design and study population characteristics. J Urol. 2010; 184: 274-9.
6. Craig JC, Simpson JM, Williams GJ, et al. Antibiotic prophylaxis and recurrent urinary tract infection in children. N Engl J Med 2009; 361: 1748 - 59.

7. Montini G, Rigon L, Zucchetta P, et al. Prophylaxis after first febrile urinary tract infection in children? A multicenter, randomized, controlled, noninferiority trial. Pediatrics 2008 ; 122 : 1064 - 71 .

8. Hoberman A, Greenfield SP, Mattoo TK, et al. Antimicrobial prophylaxis for children with vesicoureteral reflux. $\mathrm{N}$ Engl J Med. 2014 19; 370: 2367-76.

9. Tekgül S, Riedmiller H, Hoebeke P, et al. EAU guidelines on vesicoureteral reflux in children. Eur Urol. 2012; 62: 534-42.

10. Peters CA, Skoog SJ, Arant BS Jr, et al. Summary of the AUA Guideline on Management of Primary Vesicoureteral Reflux in Children . J Urol 2010; 184: 1134 44.
11. Garin EH, Olavarria F, Garcia Nieto V, et al. Clinical significance of primary vesicoureteral reflux and urinary antibiotic prophylaxis after acute pyelonephritis: a multicenter, randomized, controlled study. Pediatrics. 2006; 117: 626-32.

12. Pennesi M, Travan L, Peratoner L, et al. Is antibiotic prophylaxis in children with vesicoureteral reflux effective in preventing pyelonephritis and renal scars? A randomized, controlled trial. Pediatrics. 2008;121:1489-94.

13. Hari P, Hari S, Sinha A, et al. Antibiotic prophylaxis in the management of vesicoureteric reflux: a randomized doubleblind placebo-controlled trial. Pediatr Nephrol. 2015; 30: 479-86. 